\title{
Secret et tension narrative chez Modiano et Le Clézio : entre témoignage et créativité
}

\author{
Adina Balint-Babos \\ Université Trent
}

Pourquoi parler de secret dans une réflexion qui tente de penser les relations entre témoignage littéraire, faux témoignage et créativité? On présume que chacun peut donner une réponse à cette question. Les représentations du secret frôlent la mort et la difficulté d'en témoigner : la fuite, le nondit, la clandestinité. L'exil aussi. Si l'on a un secret, cela veut dire qu'il y a un danger, une urgence ou une menace de mort qu'on essaie d'esquiver. Avoir un secret veut dire aussi que l'on se sent piégé devant la réalité, que l'on choisit un moyen contourné d'exprimer une crainte, une façon autre de lancer un appel : une distance, un temps de réflexion. 
Penser le secret signifie également se confronter à une forme de mémoire. La démarche créatrice des descendants des victimes ou des survivants de la Shoah approche le passé de manière indirecte, moins par le souvenir puisé dans les camps de la mort que par l'imaginaire. Se pose donc la question du faux témoignage comme travail de créativité, en tant que transfiguration poétique de l'expérience de la guerre. La reconstruction littéraire s'appuie sur des récits - émanant des parents, des proches, des survivants - et non pas sur une expérience vécue. Le jeu du possible et une certaine liberté de parole et de pensée interviennent dans ce processus du faux témoignage, si l'on nomme ainsi la venue à l'œuvre des écrivains de deuxième génération, œuvre qui se trame sur fond de collecte de bribes, d'éclats, de fragments et de traces. Dans La Mémoire saturée, Régine Robin convoque le terme de «postmémoire » pour représenter « la transmission du traumatisme de guerre ou du génocide par ceux qui n'ont pas connu la guerre ou qui étaient trop jeunes pour comprendre la gravité des événements» (p.322). Pour nous, il s'agit de décoder le message que nous transmettent ces écrivains, en le portant à la limite de sa signification, et d'examiner comment l'arbitraire de la langue, le silence des blancs ou des points de suspension, l'anonymat d'un personnage, s'inscrivent en sens et tentent d'instituer une narration. Il reste la nécessité d'une méfiance radicale à l'égard de tout écrit, car lorsqu'un texte propose de dire un secret, nous sommes en droit de nous demander ce qu'il entend nous cacher en racontant. D'où aussi la quête passionnante de cet autre secret qu'il nous révélera en croyant le cacher. Déplions donc l'infinité dissimulée au cœur du récit de fiction. 
Les textes de Modiano et de Le Clézio, Dans le café de la jeunesse perdue et Ritournelle de la faim, placent tous les deux des figures du secret au centre du récit. Il s'agit du secret en tant que fuite. La fuite, non pas comme une grande aventure, comme par exemple lorsqu'on est aspiré par des paysages d'ailleurs ou poussé par le feu d'un idéal que décriraient des mots exaltants; non, la fuite, pour ces écrivains, est celle qui touche la mort et donc la difficulté de l'exprimer, de la saisir par la représentation, de la nommer. C'est la désolation que vivent les civils, et surtout deux jeunes filles, Louki chez Modiano et Ethel chez Le Clézio, sous l'Occupation à Paris pendant la Seconde Guerre mondiale. C'est la fuite devant le danger des rafles et de la déportation des juifs. Et si l'on remonte en arrière, il faut se rappeler Dora Bruder, le récit modianesque de 1997, dont le personnage éponyme pose la question de la mémoire collective face à l'Occupation ainsi que celle de la capacité du témoignage littéraire d'aborder de façon signifiante, avec force créatrice, des sujets graves de l'Histoire. Comment le romancier s'y prend-il pour sortir de l'anonymat une inconnue, Dora Bruder, lui qui n'a pas accès aux archives, à l'état civil, aux fichiers de la police? L'écrivain poursuit sa quête comme un détective : «Il faut longtemps pour que resurgisse à la lumière ce qui est effacé. Des traces subsistent dans des registres et l'on ignore où ils sont cachés et quels gardiens consentiront à vous les montrer. Ou peut-être ont-ils oublié, tout simplement, que ces registres existaient? » (Modiano, 1997, p. 15) De bribe en bribe, des pans de vie apparaissent. Déjà, en marge de la «petite histoire» personnelle, la grande Histoire fait son entrée.

Surgit donc une interrogation sur la nature des moyens dont l'écrivain dispose pour ouvrir le secret des faits et des 
ressentis, pour faire avancer son projet d'écriture sur l'arête toujours fuyante de la vérité historique et de l'imagination romanesque. Entre vrai et faux, entre témoignage documentaire et faux témoignage, à la lisière du factuel et de la créativité, le texte littéraire relance à jamais des quêtes de sens. Par-delà l'impression d'infinité possible que donne l'imaginaire du créateur, le récit se recharge en entretenant la conscience de ses limites.

\section{Pour une ligne historique}

Une première ligne, historique, lie les deux écrivains : l'histoire de l'Occupation, de la collaboration, un vécu collectif qui sert de toile de fond à des récits intimes. Cette exploration d'un événement historique marquant se lit comme un projet de connaissance de soi. Le processus relève d'une rumination scripturale du passé, régi par la fréquence d'éléments clés, posés à la fois comme des jalons dans les œuvres et comme des marqueurs qui entretiennent un certain mystère, un suspense. Des personnages, des lieux et des espaces associés à des figures validées comme biographiques sont insérés dans les récits à dominante tantôt autobiographique, tantôt romanesque. Les textes circonscrivent une histoire réelle et une dimension symbolique: la révélation d'un pan de vie intime passe nécessairement par le jeu des masques. Chez Modiano, la scène type de l'adolescence est la fugue dans sa version autobiographique qu'en présentent Dora Bruder et Un pedigree, et la fuite, dans la version romanesque qu'en développe par exemple Voyage de noces. L'une et l'autre font figure de refus; ce sont les incarnations, pour reprendre l'expression de Daniel Sibony, d'un «entre-deux» problématique, en conflit avec 
l'origine et la société : une société offrant comme seule alternative le statut de clandestin ou de délinquant, et une origine dont les racines juives nourrissent l'obsession de l'esquive. Ainsi pourrait s'expliquer la métaphore du déplacement en tant que cheminement entre l'origine - soit son inscription dans l'écriture - et la quête douloureuse d'une place dans la société, dans le monde. Déplacements géographiques : Louki, chez Modiano, glisse tel un fantôme dans les rues de Paris, tandis que, chez Le Clézio, la route de Paris à Nice, où la famille d'Ethel va se réfugier, se fait chemin de croix. Déplacements historiques et langagiers: chez les deux écrivains, il y a un va-et-vient incessant entre le présent et le passé1; la temporalité se défait, la nature des événements se dilue dans les mailles de la langue, se cache sous des échos, des faux-semblants; pas de fil narratif linéaire, pas de transparence du témoignage non plus. Et aussi déplacements psychiques et poétiques: le vécu biographique imprègne le contenu des textes, ce qui conduit au brouillage des frontières génériques. Fiction ou autofiction? Roman ou récit de soi? L'écriture cherche à repérer comment, souterrainement, s'inscrivent pour chaque sujet-personnage, dans sa langue, les effets de sens de son trauma, de son désir.

$\mathrm{Au}$ demeurant, cette question de la quête existentielle entraîne une réflexion plus large, celle du pouvoir du témoignage littéraire (voir Blanckeman et Millois). Par le sujet et la portée de leurs textes, Le Clézio et Modiano mettent en question ce que peut la littérature aujourd'hui. En même temps, ils donnent à penser la posture de l'écrivain, sa part de

${ }^{1}$ Les mots du narrateur de Dora Bruder sont révélateurs : «J'ai l'impression d'être tout seul à faire le lien entre le Paris de ce temps-là et celui d'aujourd'hui. » (p. 51) 
responsabilité, son rôle partagé d'acteur, d'observateur-témoin, de passeur ou de traducteur vrai ou mensonger du monde. Leurs textes nous conduisent également à réfléchir à ce qu'est écrire : témoigner, tisser des mots dans l'espoir que ceux-ci auront un impact sur la réalité? Ou faire passer « des appelsrappels » (Sibony, 1991, p. 181) de la part des vivants et des morts? Donner du poids à la reconnaissance? Souvent, la frontière entre la posture de l'écrivain témoin et celle du passeur s'avère imperceptible. Malgré tout, les récits de Modiano et de Le Clézio tissent un témoignage subjectif sur la Seconde Guerre mondiale. Paradoxalement, c'est par le détour de la créativité en ce que celle-ci a d'invention romanesque, de fausse vraisemblance, que le fait historique acquiert sa plus grande vérité. Chemin faisant, ces textes soulèvent aussi le défi de la mémoire fragmentaire, de l'oubli et, certes, la question de l'authentique de la représentation. Comment revendiquer pour une œuvre de fiction l'authentification de la réalité? Or, à convoquer Proust, pour qui «la vraie vie, la vie enfin découverte et éclaircie, la seule vie par conséquent réellement vécue, c'est la littérature. Cette vie qui en un sens, habite à chaque instant chez tous les hommes aussi bien que chez l'artiste. Mais ils ne la voient pas, parce qu'ils ne cherchent pas à l'éclaircir. » (p. 487), l'on est conduit à faire confiance au pouvoir des écrivains d'ouvrir et d'inscrire de nouvelles vies, des vies autres que les leurs, autres que les nôtres. Ici encore surgit l'importance de l'immobilité féconde, la portée de la quête d'une forme-sens esthétique à partir d'un vécu singulier.

Notons ensuite, chez les deux écrivains, le désir de faire passer une histoire familiale ou celle d'un groupe de gens qui se connaissent. Dans Dans le café de la jeunesse perdue et dans Ritournelle de la faim, les allées et venues entre un événement 
collectif et un événement intime interrogent le sens de la crise dans laquelle se trouvent l'homme et la société moderne : crise du modèle familial, crise identitaire, mal-être social et psychologique. Songeons à Louki, chez Modiano : cette fille erratique fuit, hormis la société, sa propre mère; elle vit dans l'anxiété continuelle de la saisie et de la des-saisie d'une identité. Pour elle, la fuite compulsive correspond au seul espace d'une éventuelle normalité, au cas où un tel état serait rendu possible par la reconstruction du souvenir : "Mes seuls bons souvenirs sont des souvenirs de fuite ou de fugue. " (Modiano, 2007, p. 95) Quant à Ethel, elle aussi apparaît comme un personnage en crise: la grande famille est déchirée par la guerre, son identité est marquée par la rupture de «l'île de l'origine » (Le Clézio, 2008a, p. 127), Maurice. Privée de l'héritage qui devait lui revenir de son oncle - «Elle n'avait pas quinze ans, elle venait de tout perdre. » (p. 68) —, elle vit mal la fuite à Nice, où les siens s'abritent pendant l'Occupation. Elle est constamment tiraillée entre le rappel d'une enfance dorée et le poids des difficultés au moment où elle a vingt ans. Dans les deux cas, on a affaire à des personnages composites qui portent quelque chose de la biographie des écrivains, mais par-delà les tensions du vécu et de la transposition fictionnelle, ces personnages proposent une représentation de l'humanité marquée par la douleur, la hantise et la culpabilité. Chacun à sa façon, les écrivains, les jeunes filles personnages-doubles fictionnels et le monde sont habités par la crise. Que cela se traduise par la fuite, la clandestinité ou la menace de la mort, un trait leur est commun: la résistance à toute fixation, à tout mode de représentation qui n'entend pas prêter écoute à des visions intuitives, à des non-dits, aux zones d'ombres de l'intériorité. Cette difficulté à saisir par l'entremise de l'écriture caractérise 
l'événement historique de la Seconde Guerre ainsi que le récit intime. Dans son hétérogénéité, à l'instar d'une composition métisse, le texte mêle la réflexion sociologique au vécu personnel, le public au privé, jouant sur les frontières de l'autofiction. Chez les deux écrivains, on perçoit un va-et-vient constant du dévoilement à l'expression, de l'exploitation à la reconstruction, comme si le récit se jouait des démarcations entre la fiction et la réalité, comme s'il était habité par une nécessité de redéfinir son rapport au réel et, par le fait même, à la fiction.

\section{Pour une ligne ontologique}

Après la ligne historique, une deuxième ligne, ontologique qui passe par les inflexions d'une histoire personnelle -, permet de relier une fois de plus Dans le café de la jeunesse perdue et Ritournelle de la faim. Modiano et Le Clézio investissent dans leurs textes une urgence à comprendre, à se construire, à lutter contre l'oubli. Leurs écrits, qui se tissent à partir de l'appropriation romanesque de la matière biographique, relèvent de la fictionnalisation de soi, du besoin d'apprendre à connaître un passé lointain, une pré-histoire familiale et communautaire. L'entreprise littéraire est d'autant plus intéressante que les deux écrivains parlent d'événements des années quarante, auxquels ils n'ont donc pas assisté, puisque Le Clézio est né en 1940 et Modiano en 1945. Blanckeman propose l'expression « témoin visionnaire » (2009, p. 140) pour nommer le paradoxe d'un auteur qui s'apprête à déchiffrer le secret d'une filiation problématique ou de celui qui s'invente une filiation faute d'en avoir une qui serait acceptable. 
Dans Ritournelle de la faim, Le Clézio invente l'histoire de la jeune fille Ethel avant l'âge de vingt ans à partir de souvenirs, de ce qu'il croyait avoir été l'existence de sa mère et des vies d'autres femmes de l'époque, dont Nathalie Sarraute: "Ethel est un personnage composite, dans lequel il y a un peu de ma mère, un peu de Nathalie Sarraute, un peu des vieilles tantes que j'ai connues, et un peu de moi-même aussi, évidemment. » (2008b, p. 48-49) Pour la première fois, Le Clézio explore ouvertement l'ascendance maternelle dans l'espoir, à jamais relancé, de saisir un peu mieux qui il est et d'où il vient, ce qui lui fournit l'occasion de retourner à son lieu d'origine. Avec le temps qui passe, l'écrivain, semble-t-il, devient confiant d'avoir trouvé la voix juste, une pureté du ton qui dévoile quelque chose de précieux. Ce quelque chose qui, pourtant, existait depuis longtemps en lui, à l'instar du « livre essentiel » (p. 468) de Proust, qu' « un grand écrivain n'a pas, dans le sens courant, à [...] inventer puisqu'il existe déjà en chacun de nous, mais à [...] traduire » (p. 468). Et s'il est vrai que les écrivains portent en eux la matière de leurs œuvres, comment ne pas songer que la maturité rend peut-être la nécessité de cette traductiontémoignage encore plus urgente? Ce n'est sans doute pas dépourvu de signification qu'à propos de Modiano et de Le Clézio, on invoque souvent la posture de «l'écrivain passeur» (voir Roussel-Gillet et Schulte Nordholt) : ces écrivains recherchent et font passer le sens des événements qui ont marqué leur vie et, au-delà de leur personne, l'humanité tout entière. Le Clézio le reconnaît : "Je ne veux pas avoir la vérité sur ma famille. Je veux connaître les tenants et les aboutissants d'une époque.»(2008b, p.48) Voilà comment l'écrivain assume le privé comme prétexte de la connaissance plus large d'une époque. 
Pour sa part, dans Dans le café de la jeunesse perdue, Modiano remet en question la connaissance de soi, compromise par l'impossibilité douloureuse de tisser une ascendance, d'établir un lien factuel ou même symbolique avec le passé, d'où la névrose obsessionnelle de la fuite qui anime le personnage principal. Dans un contexte psychanalytique, on entrevoit Louki se battre avec la quête compulsive de ce que Sibony appelle le «don premier de tout humain : le don d'un geste qui le tienne, d'une parole qui le nomme et l'appelle » (p. 79), don qui ne lui sera finalement pas accordé: «Louki. Elle s'est jetée par la fenêtre. » (p. 147) De Dora Bruder à Dans le café de la jeunesse perdue, Modiano donne l'impression qu'il écrit depuis le sentiment tragique d'une identité fantôme, dans un cycle d'éternel retour obsessionnel, comme si l'écriture tentait de saisir ce moment fugitif où se passe quelque chose qui pourrait illuminer la signification de la vie de Louki, de Dora ou le sens de leur mort. D'une manière évidente, Louki est un être caractérisé par la fuite, par le mystère, même si plusieurs personnages se lancent à son sujet dans une enquête de détective, consacrant une bonne partie de leur temps à la saisir, à retirer des témoignages sur elle. Le récit est en effet composé d'une suite de discours à la première personne attribués à ces personnages qui évoquent la jeune femme, dont le secret, loin de se dissiper, devient au contraire encore plus épais. L'événement majeur du roman à peine nommé, jamais élucidé, son suicide, est symptomatique, chez Modiano, d'un blocage sur lequel bute la narration et qui traduit aussi, sur le plan formel, une impasse de la démarche littéraire, impasse qui se manifeste par des points de suspension, des signes d'interrogation, des répliques laconiques constituées d'un seul mot. 
Ce suicide resté dans l'ombre est également le signe latent d'une aspiration au vide, d'une sorte de fuite vers le néant qui happe l'univers romanesque de Dans le café de la jeunesse perdue. Au Condé, lieu de rencontre des personnages, on tourne en rond, on répète, on fait et refait des enquêtes à l'instar d'une spirale étourdissante qui vrille vers la mort, sans qu'il soit possible de briser la cyclothymie de ce phénomène incessant de reprise. Au fond, quelle est la part de l'écrivain dans la prolifération de cette thématique de la reprise obsessionnelle? Serait-ce le signe du décalage entre ce que Modiano a vécu et ce qu'il parvient à raconter dans les limites cadrées du récit? La reprise serait alors une façon d'affiner cet écart, de minimiser la frustration que donne la distance entre le dire - entre ce qu'on peut nommer dans des mots - et le vouloir-dire, le désir. Ces paroles du narrateur de Dora Bruder en disent long sur cet effort : " Je me rends compte aujourd'hui qu'il m'a fallu écrire deux cents pages pour capter, inconsciemment, un vague reflet de la réalité. » (p. 55) Dans ces mêmes mots se disent encore la patience et le cheminement sinueux de la venue à l'écriture, car dans ce processus, le sens dernier n'est jamais dit, le dernier parcours jamais trouvé : le point d'illumination de la réalité ou du fantasme demeure continûment mystérieux, fuyant.

S'agissant de Le Clézio, il est intéressant de rappeler un projet ambitieux de jeunesse : l'écrivain rêvait dans les années 1970 de pouvoir revenir un jour sur tous ses livres et de les écrire à nouveau avec, cette fois, le corps et l'esprit d'un âge plus avancé (voir 1997). En 2008, alors qu'il fait remarquer qu'«écrire est un métier un peu secret» (p.47), son projet d'autrefois lui apparaît désormais impossible, car soumis à une chronologie irréversible. Il ne sera pas réalisé, mais ce qui 
restera dans l'esprit des lecteurs, c'est la passion de Le Clézio pour «la langue comme un être vivant» (De Cortanze, p. 69), cette langue qui bouge, change, s'échappe, se crée, et que l'écrivain a envie de reprendre, d'œuvrer comme un artisan. À noter que le «projet » assez extravagant du jeune Le Clézio est une métaphore de son désir de finition de l'écriture, une expression du besoin d'aller plus loin, de creuser plus en profondeur et d'intégrer de nouveaux apprentissages, ce qui devrait être, au fond, l'ambition de tout bon écrivain. Il représente aussi un aveu profondément humain qui s'articule à la maturité : avec les années, on voit mieux la vanité des grands décalages entre vouloir accomplir et pouvoir faire. Ritournelle de la faim comporte des microséquences qui résonnent avec la sérénité de l'âge mûr. Pour Ethel, " la vie est un sac très lourd » (p. 117), affirme le narrateur. «Elle le savait depuis l'enfance, elle connaissait chacune des pierres qui étaient entrées dans le sac. » (p. 117) Par-delà le mystère qu'enferme chaque pierre, derrière une onde de sagesse et les épreuves qu'on devine, l'échange discursif fait aussi figure de mouvance. Il apparaît comme lien entre passé et présent, entre souvenirs volontaires et involontaires, entre révélation et déjà-vu ou su. Songeons encore au narrateur proustien qui, lui non plus, ne semble pas surpris par les aléas de la vie et de la mort : "Car je comprenais que mourir n'était pas quelque chose de nouveau, mais qu'au contraire depuis mon enfance j'étais déjà mort bien des fois. » (p. 489). Il y a un lien explicite entre la révélation d'Ethel chez Le Clézio et ces mots avoués dans Le Temps retrouvé, qui créent une complicité entre le narrateur mûr, le héros enfant et l'écrivain autour de "la vérité » humaine selon laquelle les mystères de la vie et de la mort se superposent, qu'il n'y a pas de frontière étanche entre vivre et mourir : l'un nourrit l'autre 
et se nourrit de lui. Et la vie et la mort ont des sens infiniment multiples et variés : au cours d'une existence, elles prennent la place qu'on leur attribue.

Pour ce qui est de l'écrivain, il reste en effet la question de la place - car il y a place dans déplacement -, une place bienveillante où déposer un lourd héritage familial et historique. Par l'écriture, il est possible de concevoir un tel lieu. Ainsi, les deux textes de Modiano et de Le Clézio parviennent à faire sentir qu'il y a un lieu d'accueil, « une communauté inavouable » (Barthes, 2002, p. 104) et inavouée, qui existe entre les êtres vivants, leurs malheurs et leurs bonheurs. Ces récits portent aussi témoignage d'une part de notre humanité, la plus intime et la plus étrangère. Qu'il s'agisse de la guerre ou d'un parcours familial, il s'agit toujours d'un sujet qui se découvre et se construit par un acte de représentation fondé sur l'expérience vécue.

\section{Pour une ligne esthétique}

Une troisième ligne relie Dans le café de la jeunesse perdue et Ritournelle de la faim, une ligne esthétique. Par esthétique, on entend la portée du projet d'écriture chez les deux écrivains, l'inscription du réel dans la fiction, la façon dont se traduit dans le discours la thématique des récits. Il s'agit donc d'une dimension poétique de la création, par laquelle on tente de décrypter la forme de l'écriture du secret. Autrement dit, quel espace la fiction définit-elle en tant que faux témoignage lorsque l'écrivain utilise un événement personnel et historique comme base et trame de son œuvre? 
D'abord, ce sont les titres des récits qui retiennent l'attention : Dans le café de la jeunesse perdue et Ritournelle de la faim sont deux titres surprenants. Ils recèlent en effet un certain paradoxe : il y a la jeunesse, mais celle-ci est perdue; il y a une ritournelle, mais c'est la faim qui en est le sujet. Ces formules lancent déjà un appel puissant, une sorte de SOS. En même temps, elles donnent voix à l'amertume, à la tristesse d'avoir raté quelque chose. Or, malgré tout, il reste l'espoir que l'écrivain est parvenu à briser le silence, à faire sortir son personnage de l'anonymat. Rappelons encore Dora Bruder, dont le secret ne cesse d'interroger les forces et les faiblesses de la nature humaine face à l'irrépressible de l'histoire personnelle et de la grande histoire. Ce secret même devient le noyau de l'être, le lieu où se love - ne serait-ce que pendant un fragile instant - toute la puissance de la jeune fille :

J'ignorerai toujours à quoi elle passait ses journées, où elle se cachait, en compagnie de qui elle se trouvait pendant les mois d'hiver de sa première fugue et au cours de quelques semaines de printemps où elle s'est échappée à nouveau. C'est là son secret. Un pauvre et précieux secret que les bourreaux, les ordonnances, les autorités dites d'Occupation, le dépôt, les casernes, les camps, l'Histoire, le temps - tout ce qui vous souille et vous détruit — n'auront pas pu lui voler. (p. 147)

La faim suggérée dans Ritournelle de la faim est également celle du lecteur, chez qui les titres des deux récits suscitent l'envie d'aller à la rencontre des mystères discursifs, de rechercher le sens de ces expressions. On voudrait pouvoir comprendre dans quelle mesure, avec l'âge, les écrivains se sentent peut-être plus responsables vis-à-vis des urgences du monde, plus responsables aussi face au témoignage de certaines parties obscures, non-dites, de leurs vies respectives qui appellent à être racontées, écrites. L'écriture fait à nouveau œuvre de 
passeur: elle fait passer des situations, des états d'esprit et d'âme d'une génération à l'autre.

Par ailleurs, les deux titres évoquent quelque part le travail de deuil, ce processus par lequel il est possible de sortir de grandes crises - ici, la perte et ce manque qu'est la faim et que l'acte d'écrire peut accomplir. L'écriture de Le Clézio et de Modiano est ritournelle et fuite. Des métaphores qui connotent la mobilité, la lutte avec la fixité de la mortification. La ritournelle et la fuite sont à la fois des figures de déplacement musical et spatial qui ouvrent des horizons, qui tracent des points de vie. Dans Journal de deuil, Barthes pose cette question : «Écrire pour se souvenir? » (p. 125) Il y répond ainsi: "Non pour me souvenir, mais pour combattre le déchirement de l'oubli en tant qu'il s'annonce absolu. Le bientôt - "plus aucune trace", nulle part, en personne.» (p. 125) Ces paroles semblent décrire le sens, la signification des projets d'écriture chez Modiano et Le Clézio.

Pourtant, si sur le plan du contenu, à maintes reprises, on note des passerelles entre Ritournelle de la faim et Dans le café de la jeunesse perdue, pour ce qui est de la forme, des distinctions nettes existent entre les deux écrivains. D'abord, l'épaisseur des livres. Le texte de Le Clézio est plus long, plus élaboré et caractérisé par la prolifération des procédés artistiques: métaphores, listes de noms propres, monologues intérieurs, dessins. À tel point que par endroits, ce récit, surtout dans les parties intitulées «Conversations de salon » (p. 47, 59 et 69), rappelle le style proustien. En revanche, Modiano demeure l'adepte d'une poétique minimale, d'un art subtil de la modulation, de la sourdine, qui privilégie une économie de figures de style pour créer du sens et du rythme. La forme 
romanesque de son texte reste fondée sur l'ellipse narrative, sur le cryptage métaphorique, sur des coupures, des blancs. Malgré tout, et par-delà ces singularités qui font qu'on parle d'un mode d'expression modianesque et leclézien, ce qui nous paraît révélateur est de saisir une constance dans le style des écrivains, une cohérence de leur voix.

Certes, avec le passage du temps, avec la présenceabsence de la mort réelle et fictionnelle, on peut dire que l'écriture se déplace lentement, qu'elle s'affine. Elle traverse et croise le faux témoignage comme démarche féconde de créativité qu'il faut maintenir et travailler à la fois pour que ne cessent de se révéler les multiples secrets du langage et de l'histoire. Car, chemin faisant, la lecture, à l'instar de l'écriture, élimine le surplus du témoignage vrai ou faux, le dérisoire encombrant, pour se rapprocher encore et encore de ce point toujours fuyant de la vérité de soi et du monde.

\section{Bibliographie}

BARTHES, Roland. (2002), Comment vivre ensemble. Cours et séminaires au Collège de France (1976-1977), Paris, Seuil/Imec, coll. « Traces écrites ».

—. (2009), Journal de deuil, Paris, Seuil.

BLANCKEMAN, Bruno. (2009), Lire Patrick Modiano, Paris, Armand Colin, coll. «Écrivains au présent».

- et J.-C. MILLOIS. (2004), Le Roman français aujourd'hui : transformations, perceptions, mythologies, Paris, Prétexte. 
DE CORTANZE, Gérard. (2009), J.M.G. Le Clézio, Paris, Ina Cultures France.

LE CLÉZIO, Jean Marie Gustave. (1997), Entretiens avec Olivier Germain-Thomas diffusés sur France Culture.

-. (2008a), Ritournelle de la faim, Paris, Gallimard, coll. «RF ».

-. (2008b), "J'écris pour essayer de savoir qui je suis », Lire : spécial Le Clézio, no 370, p. 48-49.

MODIANO, Patrick. (2007), Dans le café de la jeunesse perdue, Paris, Gallimard, coll. « NRF ».

—. (1997), Dora Bruder, Paris, Gallimard, coll. « NRF ».

—. (2005), Un pedigree, Paris, Gallimard, coll. « NRF ».

—. (1980), Voyage de noces, Paris, Gallimard, coll. « NRF ».

PROUST, Marcel. (1989), Le Temps retrouvé, dans À la recherche du temps perdu, Paris, Gallimard, coll. «Bibliothèque de la Pléiade », t. IV.

ROBIN, Régine. (2003), La Mémoire saturée, Paris, Stock, coll. «Un ordre d'idées ».

ROUSSEL-GILLET, Isabelle. (2007), «Le Clézio, passeur au monde : l'écriture et le passage des seuils », Nouvelles Études Francophones, no 2, vol. 22, p. 152-163.

SCHULTE NORDHOLT, Annelies. (2007), «Dora Bruder: le témoignage par le biais de la fiction », dans John E. Flowers, Patrick Modiano, Amsterdam, Rodopi, p. 75-87.

SIBONY, Daniel. (1991), L'Entre-deux. L'origine en partage, Paris, Seuil, coll. « Points ». 
—. (2008), La Haine du désir, Paris, Christian Bourgois, coll. « Titres ».

\title{
Résumé
}

Les textes de Modiano et de Le Clézio, Dans le café de la jeunesse perdue (2007) et Ritournelle de la faim (2008), placent au centre des récits le secret en tant que fuite. Chez ces deux écrivains, la fuite face aux rafles des juifs dans le Paris de l'Occupation donne à penser les rapports entre témoignage littéraire, faux témoignage et créativité, en posant la question du vécu et de la transfiguration fictionnelle, ainsi qu'une interrogation sur la posture de l'écrivain et les frontières des genres. La figure du déplacement apparaît comme le fil conducteur d'une lecture qui tente de décrypter un pan de la poétique de création modianesque et leclézienne à la croisée de trois dimensions d'interprétation: historique, ontologique et esthétique.

\begin{abstract}
The narratives of Modiano and Le Clézio, Dans le café de la jeunesse perdue (2007) and Ritournelle de la faim (2008), display secret as taking the flight to save one's life during the World War II in the Occupation Paris. Representations of Jewish raffles lead us to reflect upon the link between literary testimonials, fake testimonials and creativity as well as upon life and fiction. This also allows us to inquire the figure of the writer and the borders of literary genres. The article attempts to understand the poetics of creation in Modiano and Le Clézio at the crossroads of three dimensions of analysis: historical, ontological and aesthetic.
\end{abstract}

